\title{
Unilateral Femoral Head Osteonecrosis Caused by Low- Dose Oral Corticosteroid Used for Secondary Adrenal Insufficiency From Idiopathic Hypopituitarism
}

\author{
Muhammad Rajib Hossain a, c, Saeid Karandisha, Rupesh Khanala, \\ Prem Raj Parajuli ${ }^{\mathrm{b}}$, Azad Mojahedi ${ }^{\mathrm{a}}$
}

\begin{abstract}
Osteonecrosis, commonly known as avascular necrosis (AVN) of bone, is one of the universally recognized side effects of high-dose steroid and commonly involves femur head leading to significant morbidity. But AVN of femur head due to low-dose oral corticosteroid and relatively shorter span of time is a rare occurrence. We report a case of a 41-year-old woman with hypopituitarism who developed right-sided AVN while on a little physiological replacement dose of oral hydrocortisone used only for 7 months for secondary adrenal insufficiency from idiopathic hypopituitarism. Patient was diagnosed with MRI at early stage and managed with lowest possible physiological preparation of hydrocortisone and alendronate and eventually prevented from hip collapse. There should be a high clinical index for patients with any dose of steroid to recognize early, and prevent fracture and ultimately replacement.
\end{abstract}

Keywords: Osteonecrosis; Avascular necrosis; Steroid side effects; Femoral head avascular necrosis; Hypopituitarism; Steroid-dependent; Steroid-induced bone dystrophy

\section{Introduction}

Osteonecrosis or avascular necrosis (AVN) of femoral head has been associated with traumatic and numerous atraumatic conditions that lead to significant morbidity. Among them use of glucocorticoids and excessive alcohol intake are associated with more than $80 \%$ of atraumatic cases [1]. The process is most often progressive, resulting in joint destruction within a few months to 2 years in the majority of patients $[2,3]$. In the United States, there are an estimated 20,000 - 30,000 patients newly diagnosed each year [4], with even larger numbers

Manuscript submitted June 6, 2017, accepted June 19, 2017

aDepartment of Medicine, Interfaith Medical Center, Brooklyn, NY, USA ${ }^{b}$ Reading Health System, Reading, PA, USA

${ }^{\mathrm{c} C}$ Corresponding Author: Muhammad Rajib Hossain, Department of Medicine, Interfaith Medical Center, Brooklyn, NY, USA. Email: rajibssmc@gmail.com

doi: https://doi.org/10.14740/jmc2857w worldwide, and it is the underlying diagnosis in approximately $10 \%$ of all total hip replacements (THRs) in the United States [5]. Patients who are receiving prolonged high doses of glucocorticoids are at significant risk of developing AVN. However, this risk appeared to be low in patients receiving doses of prednisone less than $15-20 \mathrm{mg} /$ day [6]. The case we presented here is an example of such case who developed AVN while on very low dose of steroid and relatively shorter period of time. Early diagnosis is necessary to prevent progression, collapse and eventually the need of hip replacement.

\section{Case Report}

A 41-year-old average built African American female with persistent hot flushes, irregular menstrual cycle, extreme fatigue and cold intolerance was evaluated for early menopause and thyroid dysfunction. Physical exam was only remarkable for delayed ankle reflex, normal development of secondary sex characters. She did not have galactorrhea. She is married with children and lives with family. She does not smoke cigarette or drink alcohol at regular basis. She was eventually diagnosed

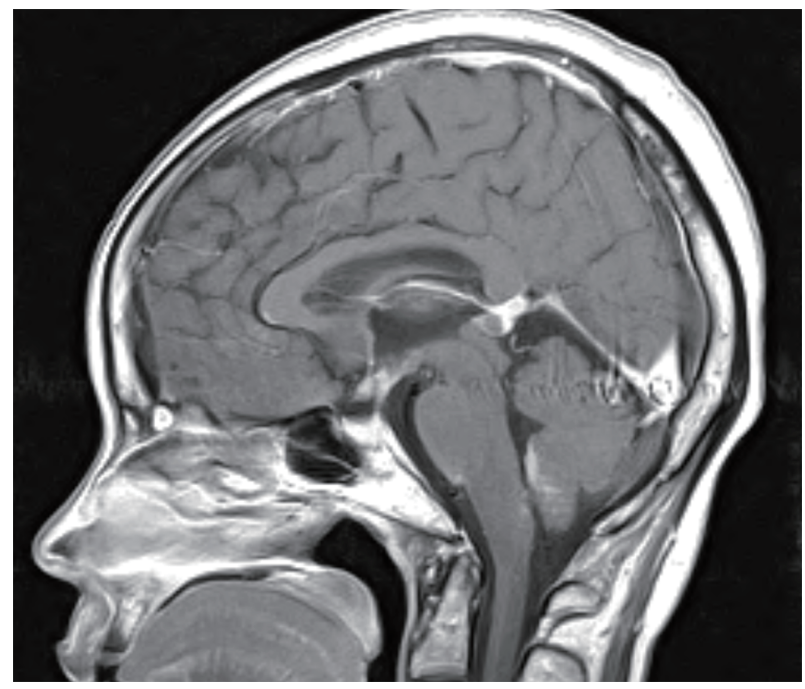

Figure 1. MRI of brain and pituitary with and without gadolinium did not show any tumor or other cranial abnormalities. 


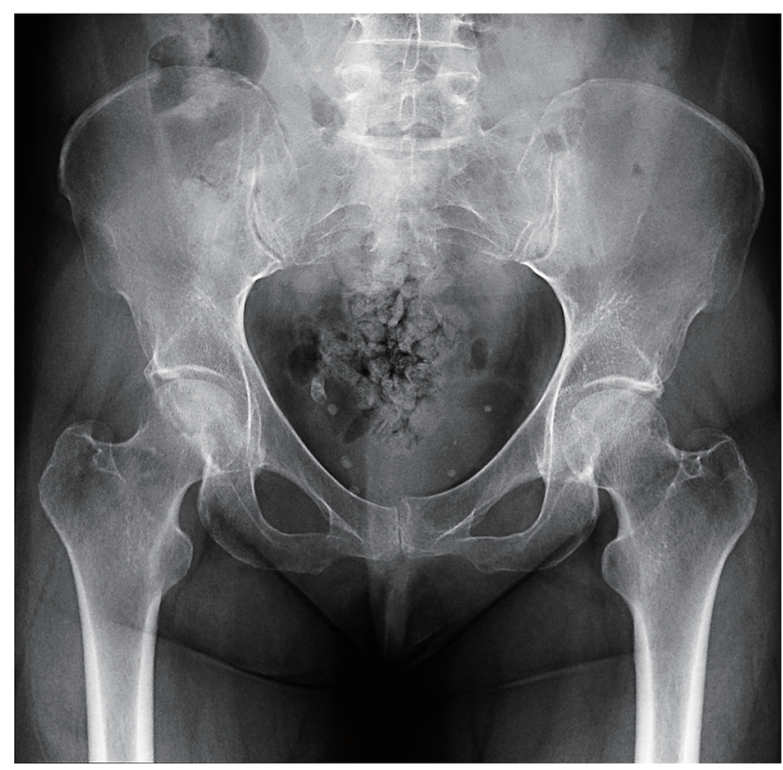

Figure 2. X-ray of bilateral hip and pelvis shows completely normal bilateral femoral head.

with partial hypopituitarism after an extensive metabolic workup which revealed low ACTH $(4.5 \mathrm{pg} / \mathrm{mL})$, FSH $(0.9$ $\mathrm{mIU} / \mathrm{mL}), \mathrm{LH}(0.1 \mathrm{mIU} / \mathrm{mL})$ and TSH $(0.421 \mu \mathrm{IU} / \mathrm{mL})$ along with low AM cortisol $(1.1 \mu \mathrm{g} / \mathrm{dL})$ and free T4 $(0.66 \mathrm{ng} / \mathrm{dL})$, estradiol $(<5 \mathrm{pg} / \mathrm{mL})$, IGF-1 $(24 \mathrm{ng} / \mathrm{mL})$, but normal prolactin level $(13.6 \mathrm{ng} / \mathrm{mL})$ which points out to a central cause of insufficiency. MR of brain and pituitary with and without gadolinium was normal (Fig. 1) and did not show any tumor or other

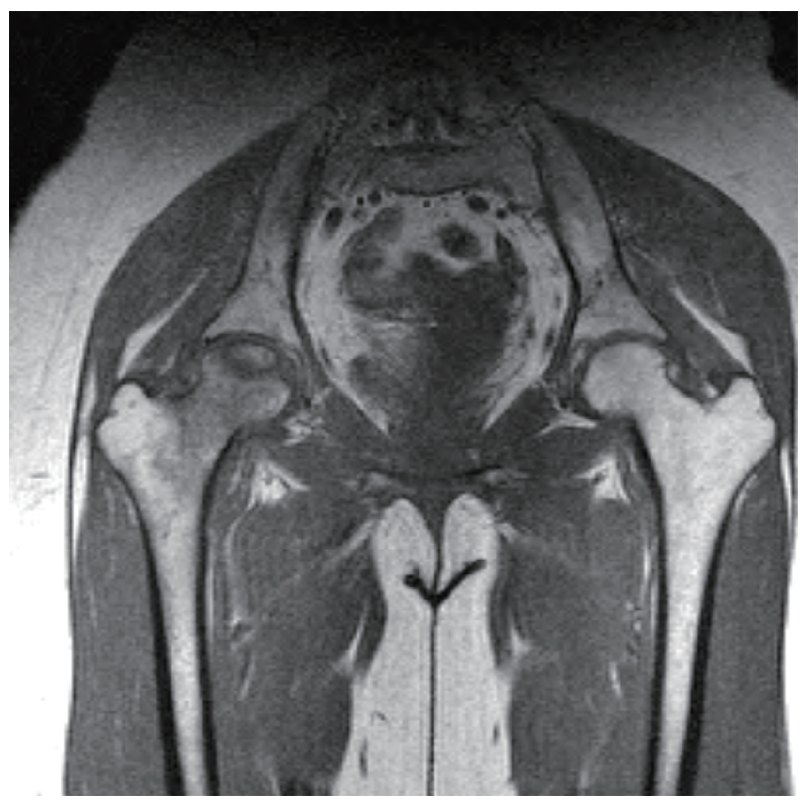

Figure 3. Coronal view of MRI of right hip demonstrates a wedgeshaped subchondral focus with hypoindense peripheral band and central marrow fat signal intensity compatible with avascular necrosis. Marrow edema of right femoral head and neck. Small joint effusion right hip.

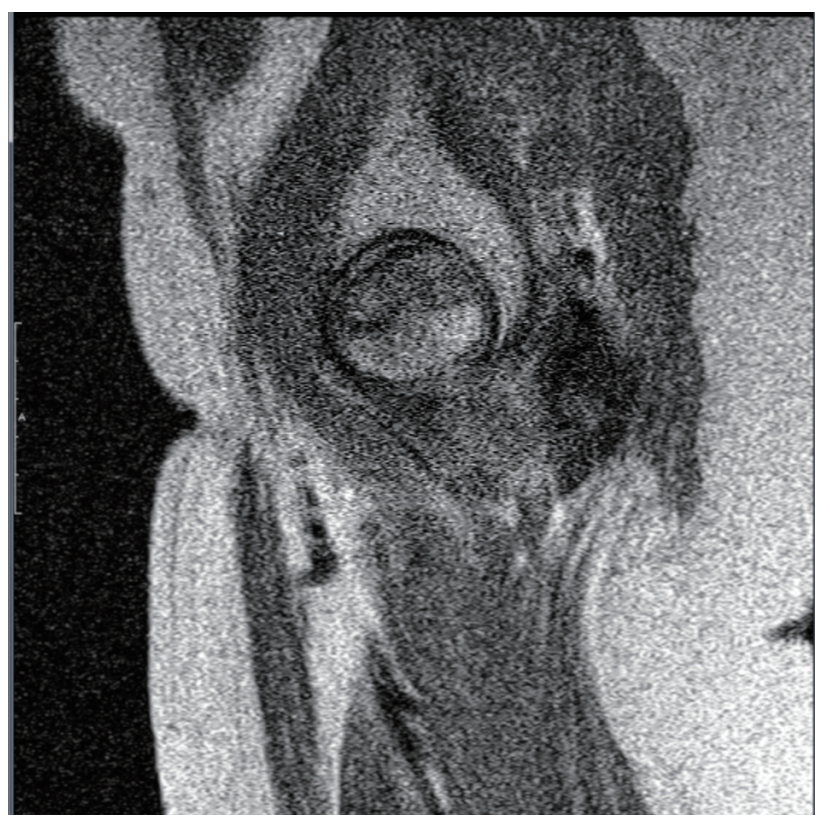

Figure 4. Sagittal view of proton density MRI of the right hip in the same patient confirms the wedge-shaped subchondral area of AVN present at the anterior-superior femoral head.

abnormality. She did not have any history of traumatic brain injury (TBI), cranial surgery, radiation, subarachnoid hemorrhage or postpartum hemorrhage. Infectious and infiltrative causes including sarcodidosis, tuberculosis, hemochromatosis, and histoplasmosis were also ruled out as patient did not have any compatible clinical, laboratory and radiographic manifestations. Pituitary hormone deficiency due to gene mutations was also considered very unlikely due to age of manifestation of disease and pattern of hormone deficiency and finally was considered as idiopathic hypopituitarism.

Her symptoms improved with oral hydrocortisone $(15 \mathrm{mg}$ $\mathrm{PO}$ in am and $10 \mathrm{mg}$ in pm initially) and thyroxine ( $88 \mu \mathrm{g}$ PO daily). Patient was stable without any adrenal crisis.

Seven months after receiving the replacement dose of steroid, patient experienced insidious onset of right hip pain which became severe enough to limit her functional capacity. Initial X-ray was normal (Fig. 2). Due to high suspicious and risk factor, MRI of the right femoral head was done which demonstrated a wedge-shaped subchondral focus with hypointense peripheral band and central marrow fat signal intensity compatible with avascular necrosis (Figs. 3 and 4). MRI of left hip was completely normal. Patient underwent bone density survey which was compatible with osteoporosis of the lumbar spine and bilateral hips. Patient was screened for sickle cell disease as a cause of osteoporosis which came back negative.

After the diagnosis, patient was evaluated by orthopedics for hip replacement, started alendronate, calcium, vitamin $\mathrm{D}$ and oral hydrocortisone at minimum dose of $5 \mathrm{mg} \mathrm{PO}$ in morning and $2.5 \mathrm{mg}$ in evening as it is considered to be the more physiological preparation of glucocorticoids. Thyroxin dose was also reduced to $55 \mu \mathrm{g}$. Patient is currently ambulating with cane when needed and stable without having any adrenal 
crisis.

\section{Discussion}

High-dose use of corticosteroids is the most common cause of nontraumatic avascular necrosis [7]. The exact reason is unknown, but it is believed due to a complex interplay and imbalance of bone resorption and formation, impairment of vasculature within bone and apoptosis.

There is no dose cutoff for the occurrence of AVN after steroid use. Powell et al [8] reviewed 66 cases of steroidinduced AVN and found that the most commonly associated administered route included intravenous steroids followed by oral and rarely intramuscular, intra-articular injections and inhaled steroids. Karkoulias et al [9] reported aseptic femoral head necrosis in a patient receiving long courses of inhaled and intranasal corticosteroids. The occurrence of AVN after steroid use appears to be dose-related. But it appears in the majority of the studies that patients are at increased risk of AVN who receive $>20 \mathrm{mg}$ /day of prednisone (which is equivalent of 80 $\mathrm{mg}$ of hydrocortisone) which is much higher than the dose of our patient [6]. However, another study done by Dimant et al [10] failed to find a correlation between peak dose, duration or cumulative dose of steroid and osteonecrosis.

The occurrence of osteonecrosis with such a low dose of corticosteroid was surprising. Tokuhara et al [11] examined the relationship between osteonecrosis and steroid dose in white rabbits and they observed that low levels of steroid metabolizing hepatic activity may increase responsiveness to steroids and further risk of steroid-induced osteonecrosis even with low steroid dose.

In our patient, menopause can be an added factor to steroid in occurrence of osteonecrosis. She was evaluated by GYN but was not considered hormone replacement therapy after discussing risk and benefit.

It is important to look for AVN in any patient with hip pain on any dose of steroid. Early detection of hip AVN is critical, as all treatments geared towards preservation of the femoral head are more successful early in the course of the disease. Management by stopping steroid can be challenging in many cases due to adrenal crisis or need to maintenance steroid to maintain remission or control disease. In such cases, standard management is done by reducing steroid to the lowest possible dose. Similar in our patient, it was not prudent to stop steroid completely to prevent fatal crisis. It is recommended to continue glucocorticoids but lowest possible dose.

Studies also looked at the use of bisphosphonates (alendronate) in preventing osteonecrosis. McGrory et al [12] found in their study that bisphosphonates is helpful in delaying collapse of the femoral head and thereby delaying need of aggressive hip replacement procedure.

\section{Conclusion}

Early recognition and management for AVN of bone in patients with any dose of steroid can prevent further complications.

\section{Conflicts of Interest}

We declare that there are no conflicts of interest regarding the publication of this paper.

\section{Author Contributions}

We, all authors certify that we all participated sufficiently in the intellectual content and each author has reviewed the final version of the manuscript and approves it for publication.

\section{References}

1. Mont MA, Hungerford DS. Non-traumatic avascular necrosis of the femoral head. J Bone Joint Surg Am. 1995;77(3):459-474.

2. Rajpura A, Wright AC, Board TN. Medical management of osteonecrosis of the hip: a review. Hip Int. 2011;21(4):385-392.

3. Mont MA, Jones LC, Hungerford DS. Nontraumatic osteonecrosis of the femoral head: ten years later. J Bone Joint Surg Am. 2006;88(5):1117-1132.

4. Moya-Angeler J, Gianakos AL, Villa JC, Ni A, Lane JM. Current concepts on osteonecrosis of the femoral head. World J Orthop. 2015;6(8):590-601.

5. Mankin HJ. Nontraumatic necrosis of bone (osteonecrosis). N Engl J Med. 1992;326(22):1473-1479.

6. Zizic TM, Marcoux C, Hungerford DS, Dansereau JV, Stevens MB. Corticosteroid therapy associated with ischemic necrosis of bone in systemic lupus erythematosus. Am J Med. 1985;79(5):596-604.

7. Weinstein RS. Glucocorticoid-induced osteonecrosis. Endocrine. 2012;41(2):183-190.

8. Powell C, Chang C, Naguwa SM, Cheema G, Gershwin ME. Steroid induced osteonecrosis: An analysis of steroid dosing risk. Autoimmun Rev. 2010;9(11):721-743.

9. Karkoulias K, Charokopos N, Kaparianos A, Sampsonas F, Tsiamita M, Spiropoulos K. Aseptic femoral head necrosis in a patient receiving long term courses of inhaled and intranasal corticosteroids. Tuberk Toraks. 2007;55(2):182-185.

10. Dimant J, Ginzler EM, Diamond HS, Schlesinger M, Marino CT, Weiner M, Kaplan D. Computer analysis of factors influencing the appearance of aseptic necrosis in patients with SLE. J Rheumatol. 1978;5(2):136-141.

11. Patton PR, Pfaff WW. Aseptic bone necrosis after renal transplantation. Surgery. 1988;103(1):63-68.

12. McGrory BJ, York SC, Iorio R, Macaulay W, Pelker RR, Parsley BS, Teeny SM. Current practices of AAHKS members in the treatment of adult osteonecrosis of the femoral head. J Bone Joint Surg Am. 2007;89(6):11941204. 